\title{
Combination of Myoware Muscle Sensor, Bluetooth Module and Analog Receiver
}

\author{
Dmitry Andreevich Artemyev ${ }^{1}$, Ilsiyar Ildarovna Bikmullina ${ }^{2}$ \\ ${ }^{1}$ Student (Department of Quality Management), Engineering Institute, Kazan Federal University, Russia.

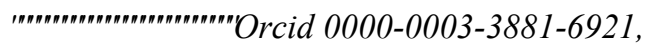 \\ ${ }^{2}$ Associate Professor (Department of Quality Management), Engineering Institute, Kazan Federal University, Russia. \\ Orcid 0000-0002-0194-2687, ID Scopus 57213153374,
}

\begin{abstract}
The article focuses on the lack of the ability to transmit the readings from the Myoware electromyography sensor via an analog signal. They described the method with the connection of a Bluetooth module to solve this problem, since there is a direct need to control, for example, robotic manipulators or a robot arm. As presented in the article, this may be demanded by a large number of the population using a convenient mobile device. They described the method of its use to solve the problems with reading taking from muscles and their further receiving on a smartphone using a Bluetooth module. They described the code development method, as well as the diagram of sensor connecting to the Arduino board. Due to the uniqueness of the measurement, as well as the capabilities of the Myoware muscle sensor, an attempt is made to deviate from the standard procedure measuring the electrical activity of muscle fibers. The code has a very simple form and is understandable to any person who is just learning to program. Thanks to the demonstration of the ports to which the sensor and modem are connected to the microcontroller, it is easy to assemble the entire model. The obtained result will help to advance in the robotic and medical fields.
\end{abstract}

Keywords: Myoware Muscle Sensor, Electromyography, Arduino Controller, Surface Electromyography Sensors

\section{INTRODUCTION}

The article is devoted to the creation of symbiosis of the Myoware muscle contraction sensor and the Bluetooth module. This approach is focused on the system design process. The article discusses the development method based on the $\mathrm{C}++$ programming language in Arduino software, which is used to develop simple automation and robotics systems. The application program provides the data on muscle contractions in the range from 0 to 1023 . The test bench is used to receive an analog signal on any smartphone with Bluetooth.

The object of the study is the Myoware muscle sensor and the Bluetooth hc-06 module.

Purpose of the study. To receive an analog signal from the Myoware sensor using a Bluetooth module.

This increases the possibilities for the development of new projects, which make the process of data obtaining much easier, and will also allow further use of our method for the development of controlled manipulators using muscle contractions via the bluetooth transmission module, as well as for medical institutions.

At the moment, there have been no analogues in Russia concerning the solution for analog signal data receiving from myoware muscle sensors.

\section{METHODS AND MATERIAL}

\section{II.I Comparative analysis of software}

We chose the $\mathrm{C}++$ programming language, namely Arduino. So why did we settle for Arduino?

At the moment, many other microcontrollers and microprocessor devices are known for various hardware programming: Parallax Basic Stamp, Netmedia's BX-24, Phidgets, MIT's Handyboard and many others. All of these devices have similar functionality and they are designed to free the user from deep immersion in the small details of the microcontroller internal structure, they provide him with a simple, as well as convenient programming interface. The process of working with microcontrollers in Arduino is also simple, but there is a number of advantages in comparison with other systems for teachers, students and radio amateurs:

- Low cost. As compared to other similar hardware platforms, you can easily order the boards from China at a relatively cheap price: ready-made Arduino modules cost no more than 3000 r., and the ability to assemble the board manually allows you to save money as much as possible and get Arduino at the lowest price.

- Cross-platform. Arduino software runs on such operating systems as Windows, Macintosh OSX and Linux. At the same time, most of these systems are aimed to work in Windows only.

- Simple and convenient programming environment. Programming in Arduino is clear and straightforward for beginners, but Arduino is flexible enough for advanced users. It is based on the Processing programming environment, which can be easy and convenient for teachers. The students learning Processing programming will be able to master Arduino easily.

- Extensible open source software. Arduino software is open source, thanks to which high-level programmers can modify 
and supplement it. $\mathrm{C}++$ libraries expand the programming possibilities. Based on the fact that it is based on the AVR C language, all users who want to understand the technical details can easily switch from the Arduino language to $\mathrm{C}$ or import the sections of AVR-C code into the Arduino program directly.

Expandable open hardware. Arduino is based on the Atmel ATmega8 and ATmega168 microcontrollers. All Arduino module diagrams are published under the Creative Commons license, so that absolutely all engineers and developers can create their own versions of devices based on existing ones. And even ordinary users can assemble Arduino prototypes for a better understanding of their work and to save money [1].

\section{II.II Description of software}

To diagnose the condition, study the dynamics of the wrist joint, as well as to control exoskeletons, the value of muscle activity can be used as primary information [2]. Determination of muscle activity - the total biopotential of muscles, including in real time, can be carried out using a non-invasive procedure - surface electromyography. As for the wrist joint, it is advisable to measure the biopotentials of four muscles that implement the movement of this joint in the planes of extension-compression and adductionabduction [3].

Electromyography (EMG) is the method of muscle fiber electrical activity measurement during activation [4]. Each time your muscle moves, your muscle fibers are activated by the signals transmitted from the brain to the muscle fibers through the central nervous system and motor neurons. There are usually more negative charges inside the muscle cell than outside, but the positive action potential spreads along the entire length of the muscle fiber when the muscle contracts [5]. EMG includes the measurement of this action potential passing along the muscle fiber at 2-6 m/s [6]. Such transient differences in electrical charges can be detected with two (or more) electrodes placed along this route, while amplifying the difference and suppressing the overall voltages (through the process called differential amplification). Thus, EMG can show the muscle contraction intensity, duration and order [4-7].

Surface (global, interference, total, cutaneous) electromyography is a non-invasive research method that allows to evaluate the total bioelectrical activity of muscles at rest and during motor action performance of varying coordination complexity by recording the bioelectrical activity with surface electrodes installed cutaneous over the motor point of the muscle, followed by signal analysis on the electromyograph [8-10].

The Myoware muscle sensor is shown on Fig. 1, the sensor is small, so it is very convenient to use.

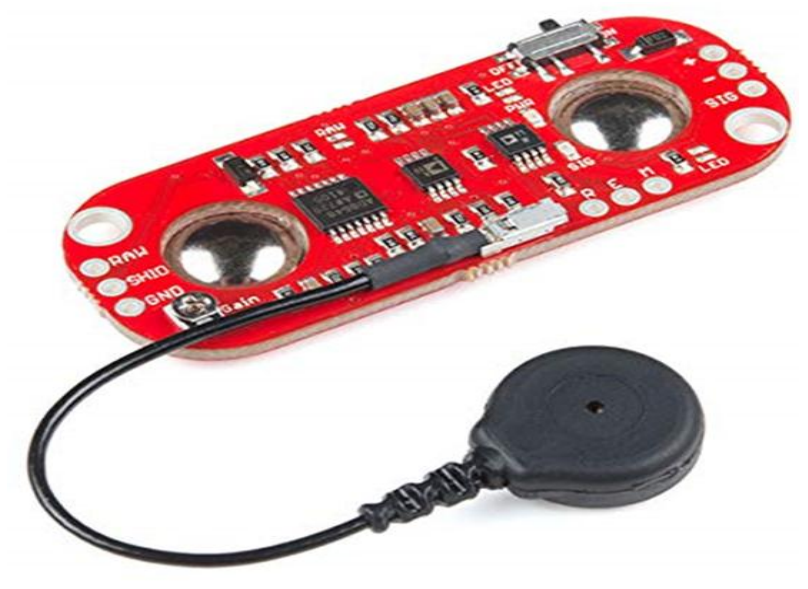

Figure 1. Myoware muscle sensor

Also the official website of the manufacturer presents the form of signals obtained during measurements, they are shown on Fig. 2

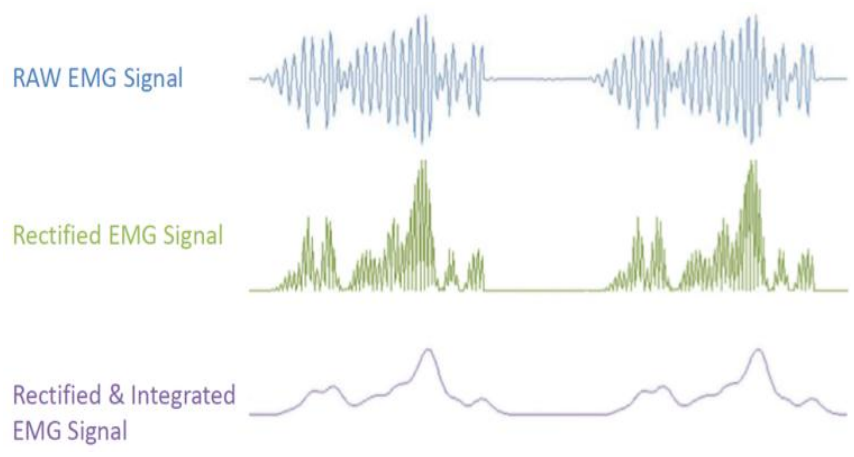

Figure 2. The signal from the Myoware muscle sensor

The sensor has three connection points to the body. Each measuring channel consists of three electrodes, two of which measure biopotentials, and the third serves as a relative ground $[11,12]$. Some of the best attachment places are the following: forearm, neck, biceps, calves [13]. The connection method is shown on Fig. 3.

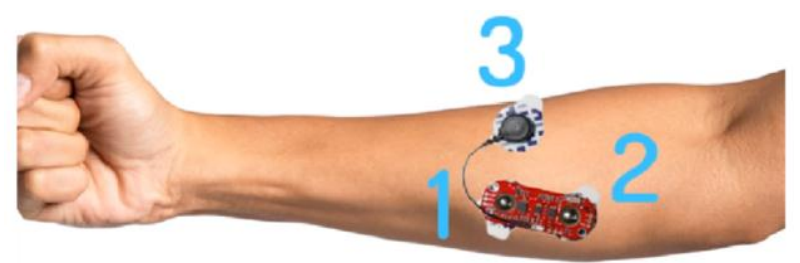

Figure 3. The way of connecting the myoware muscle sensor to the forearm muscles

Figure 4 shows the electrical characteristics of the Myoware mucsle sensor from the developer's official website [14]. 
Table I. Electrical characteristics

\begin{tabular}{|c|c|}
\hline \multicolumn{1}{|c|}{ Specifications } & Value \\
\hline $\begin{array}{c}\text { Supply voltage } \mathrm{V}_{\mathrm{a}} \mathrm{V} \\
\text { kOhm }\end{array}$ & $3.3-5$ \\
\hline $\begin{array}{c}\text { Gain adjustment potentiometer, } \\
\text { Input resistance, GOhm }\end{array}$ & 110 \\
\hline $\begin{array}{c}\text { Supply current, mA } \\
\text { (CMRR) }\end{array}$ & 110 \\
\hline $\begin{array}{c}\text { Common Mode Rejection Ratio } \\
\text { Input displacement, pA } \\
\text { Output } \\
\text { voltage Worked out } \\
\text { EMG signal, V }\end{array}$ & $0-\mathrm{V}_{\mathrm{s}}$ \\
\hline Size, cm & $5,23-2,07$ \\
\hline Estimated cost, rub. & 2140 \\
\hline
\end{tabular}

Bluetooth module HC-06 (Fig. 4) for wireless device control. Able to work with any USB Bluetooth adapter, the data transfer rate is $9600 \mathrm{bps}$, the range is up to 10 meters.

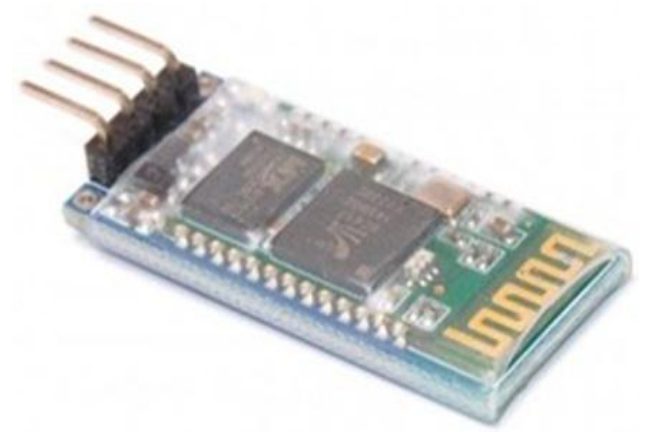

Figure 4. Bluetooth HC-06 module

\section{RESULTS}

Based on the abovementioned comparative analyzes of software, the type of controllers, as well as a specific type of controllers from one manufacturer, we can conclude that from the totality of the functions we require, the Arduino microcontroller is most suitable - the inexpensive cost of the board itself, as well as a large number of sensor manufacturers, which make their products for Arduino, their low cost and ease of integration into the system. As software, we chose $\mathrm{C} / \mathrm{C}++$ due to the fact that the required range of functions can ideally be performed by this language, as well as the ease of integration with the libraries of the Python programming language, as well as a huge native library, ease and clarity of the language. MEGA was chosen as a specific Arduino board, since it has a large number of ports, higher RAM and internal memory. All this will be required in order to ensure the stable simultaneous operation of our sensors.
In the course of the research, we identified the necessary circuit for connecting the sensor to the microcontroller, the voltage required to connect it, and the most convenient location for communication through the sensor cables to the microcontroller.

Connection diagrams were presented, unfortunately, a very convenient program for the advance construction of sensors in a single format via the "fritzing" program was not available to us because it did not contain the sensors we used, so we had to check the circuit by comparing the known information about the work sensors within the limits of voltages and other indicators. The wiring diagram was presented on the images, allowing us continue the project coding and designing with firm confidence in the project safety and its compatibility with other sensors.

The connection diagram of all Myoware and Bluetooth module to the Arduino Mega 2560 microcontroller is the following:

Bluetooth к Mega2560:

VCC - $3.3 \mathrm{v}$
GND - GND
TXD - P10
RXD - P11

Myoware to Mega2560:

$+-5 \mathrm{v}$

GND - GND

SIG - A0

The source code will take the form shown on Figure 5.

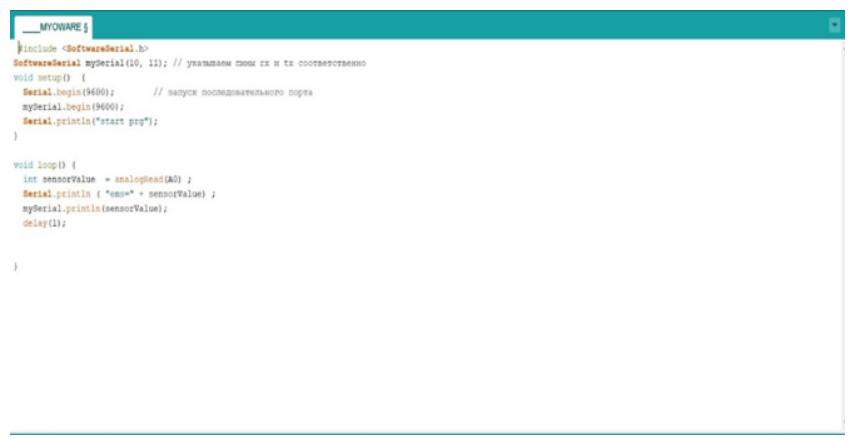

Figure 5. The code for Myoware Sensor and HC-06 Bluetooth Module

Now our code is ready, all that remains is to download the application to your smartphone, which will be able to perform the functions of a Bluetooth terminal. For these purposes the application "Bluetooth terminal was selected.

Figure 6 shows the results obtained from the Myoware muscle sensor in the "Bluetooth Terminal" application. 


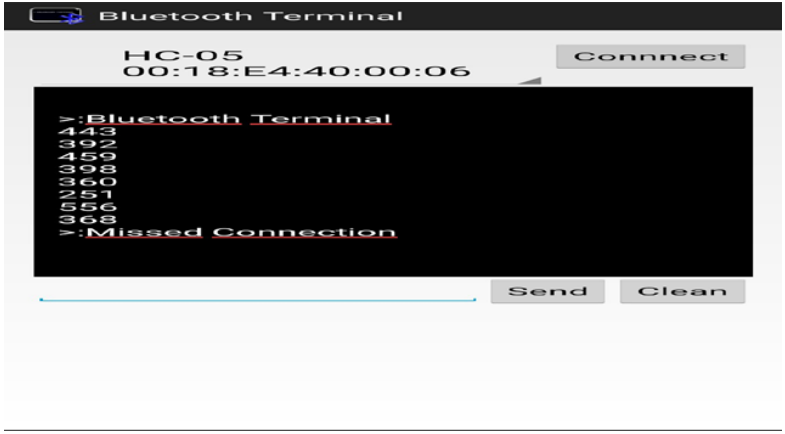

Figure 6. Myoware sensor results

In connection with the functions we required from the sensor, it was undertaken to take the sensor readings in a numerical equivalent, and not in the form of a graph, because this innovation will help us program the manipulator arm remotely through this.

\section{DISCUSSION}

Moving away from modern methods of electromyographic (EMG) data obtaining, from these large complexes for data receiving, we can say that the future lies behind this, behind the mobility of the entire system, and the way of data obtaining. Earlier, Shcherbak O.Yu., Maslennikov A.L., Zadorozhnaya N.M., [15] considered the possibility of displaying four channels of electromyography measurements in real time using four Myoware sensors, as well as the possibility of filtering by the floating average method, but all this was displayed on a computer, without the ability to transfer the resulting indicators to a smartphone. To get the results we need, we decided to leave only one sensor, due to the fact that at the moment we needed to transfer the readings to the monitor of a smartphone or any analog signal receiver, without an average indicator. Thanks to the correct construction of the circuit, the code, as well as the correct connection of the sensor and modem to the controller, we managed to achieve the set task.

The use of robotization in production is necessary to achieve the required quality of products. The use of an industrial robot gives an unlimited range of possibilities for workpiece processing. The robotic system can be used without readjustment for auxiliary operations [16-17].

\section{SUMMARY AND CONCLUSION}

In the future, it is planned to use the results obtained in the field of robotics, and in the medical field. Through the introduction of galvanic skin response sensors, as well as the sensors for pulse and the percentage of oxygen in the blood, a wide range of measurements will be obtained, thanks to which the process of taking these readings will be improved. The operation of the system will be mobile, because it is powered by a specific case with batteries, which will be able to charge right in the case, without the need to remove them.
A specific building will be designed to make the system autonomous, in order to use it by a person outside the home, a database will be built for data storage, and if the wearer wants to check previously measured readings, the access to them will be provided. Thanks to the uniqueness of the measurement, as well as the capabilities of the Myoware muscle sensor, we can move away from the standard procedure of muscle fiber electrical activity measurement. Receive readings at a distance of up to 10 meters, and the result we have obtained will help to advance in the robotic and medical fields. The code has a very simple form and will be understandable to any person who is just learning to program. Thanks to the demonstration of the ports to which the sensor and modem are connected to the microcontroller, it will be very easy to assemble the entire model.

\section{ACKNOWLEDGEMENTS}

The work is performed according to the Russian Government Program of Competitive Growth of Kazan Federal University.

\section{REFERENCES}

[1] URL: https://doc.arduino.ua/ru/about/ (reference date: 31.05.2020)

[2] Gavrilov AI, So So Tav U. Application of electromyography data in control systems of exoskeletal devices. Engineering Journal: Science and Innovation. Electron. journal. 2017, issue 6. URL: http://engjournal.ru/catalog/mech/bmech/1623.html (reference date: 05/16/2018).

[3] Palmer AK, Werner FW, Murphy D, Glisson R. Functional wrist motion: a biomechanical study. Journal of Hand Surgery. 1985 Jan 1;10(1):39-46.

[4] Hamilton N, Weimar W, Luttgens K. Kinesiology: Scientific basis of human motion. New York, NY: McGraw-Hill, 2012.

[5] Konrad P. The ABC of EMG: A practical introduction to kinesiological electromyography. 2005.

[6] Baker R, Shortland A. Electromyography. in Measuring Walking: A Handbook of Clinical Gait Analysis, R. Baker, Ed. London: Mac Keith Press. 2013"71-87.

[7] Jamal MZ. Signal acquisition using surface EMG and circuit design considerations for robotic prosthesis. Computational Intelligence in Electromyography Analysis-A Perspective on Current Applications and Future Challenges. 2012 Oct 17;18:427-48.

[8] Gekht BM. Theoretical and clinical electromyography. L.: Science; Gekht B.M. Teoreticheskaya i klinicheskaya elektromiografiya [Theoretical and clinical electromyography]. Leningrad: Nauka; 1990. 
International Journal of Engineering Research and Technology. ISSN 0974-3154, Volume 13, Number 11 (2020), pp. 3519-3523

(C) International Research Publication House. https://dx.doi.org/10.37624/IJERT/13.11.2020.3519-3523

[9] Badalyan LO, Skvortsov IA. Clinical electromyography," M: Medicine; Klinicheskaya elektromiografiya [Clinical electromyography]. Moscow: Meditsina; 1986.

[10] Aminoff, M. Electromyography in clinical practice. Addison-Wesley, 1978.

[11] Nikolaev SG. Atlas of electromyography. - 2nd ed., rev. and add. - Ivanovo: PressSto, 2015; 488 p.

[12] Nikolaev SG. Electromyography: clinical practice. Ivanovo: PressSto, 394 p, 2013.

[13] URL: arduino.ua/prod2090-nabor-myskylnogodatchika-dlya-arduino (reference date: 30.05 .2020 )

[14] URL: https://cdn.sparkfun.com/assets/a/3/a/f/a/AT04-001.pdf (reference date: 1.06.2020)
[15] Shcherbak OYu, Maslennikov AL, Zadorozhnaya NM. Surface electromyography data collection system. Problems and perspectives of student science. 2018;1(3):33-34.

[16] Khafizov II, Nurullin IG, Kashapov NF. Influence of application of robotic systems on prospects and directions of development of electroerosive processings. InIOP Conference Series: Materials Science and Engineering 2019 Jul (Vol. 570, No. 1, p. 012049). IOP Publishing.

[17] Kashapov NF, Khafizov II, Nurullin IG, Sadykov ZB. Influence of introduction of robotics on increase in efficiency of electrochemical production. InIOP Conference Series: Materials Science and Engineering 2018 Jan 1 (Vol. 412, No. 012034).

Dmitry Andreevich Artemyev, student. Fields of scientific interests: biometric sensors, robotics, quality management of robotic processes. In addition to English, speaks Czech.

Ilsiyar Ildarovna Bikmullina, the author of over 40 scientific articles, 2 research monographs, 4 certificates for a computer program. The theme of the thesis is Automatic synthesis of UML class diagrams based on domain associative relationships. The major is intelligent information systems, structural systems, robotics, computer graphics, computer vision, computer game development. 